\title{
Missense Suppression in Coprinus lagopus Associated with a Chromosome Duplication
}

\author{
By D. LEWIS \\ Department of Botany and Microbiology, University College, London $W C_{\mathrm{I}} E 6 B T$ \\ AND LORNA A. CASSELTON \\ Department of Plant Biology and Microbiology, Queen Mary College, London EI $4 N S$
}

(Received 20 August 1974; revised 30 December 1974)

\section{SUMMARY}

Amongst some 70 recessive suppressors of a met-I mutation in Coprinus lagopus, one unstable suppressor was identified. The unstable suppressor, designated sup- $6^{+}$, could be maintained on minimal medium, but was lost within $24 \mathrm{~h}$ on minimal medium containing more than 1.7 p.p.m. DL-methionine or 0.75 p.p.m. Lmethionine. Isolation of hyphal tips from the monokaryotic strain carrying sup- $6^{+}$ yielded three types of colony: the unstable parental type, the stable met-I auxotroph and a stable prototroph which was slow-growing and inhibited by methionine in the growth medium. This stable sup- $6^{+}$type was recovered with difficulty by resolving dikaryons formed between the unstable sup- $6^{+}$strain and strains carrying the wild-type allele of the suppressor gene. From sexual crosses, neither the unstable nor stable sup $-6^{+}$type segregated, only the met-I auxotrophic revertant. The unstable sup- $6^{+}$strain is thought to have an extra chromosome carrying the sup $-6^{+}$mutation. For vigorous growth the wild-type allele, sup $-6^{-}$, is indispensable and would be carried on the homologous chromosome. The selective pressures on different media account for loss of the duplicated chromosomes. The results are interpreted as missense suppression by a mutant of an indispensable tRNA.

\section{INTRODUCTION}

Many suppressor mutations are known to be mutations in structural genes for transfer RNA (see Gorini, 1970; Smith, 1972, 1973). Suppression results from mistranslation of a particular codon by the mutationally altered tRNA molecule. In bacteria the best characterized tRNA suppressor genes are those which suppress nonsense codons, but tRNA suppressors of missense mutations and frameshift mutations are also known. In all but one of the mutant suppressor tRNAs sequenced (Hirsh, I97I), there has been an alteration to the anticodon which allows the tRNA to translate a different codon (Goodman et al. 1968; Altman, Brenner \& Smith, I97I; Riddle \& Carbon, I973; Yaniv et al. 1974).

In acquiring a new translation specificity the original translation function of the mutant tRNA is generally lost. Alteration to an indispensable tRNA species is lethal in a haploid cell, but lethality can be overcome if there are two copies of the tRNA gene and only one is mutated to suppressor. This is illustrated by the haploid lethal chain terminating suppressors of Escherichia coli which were selected in a strain carrying an episome duplicating the suppressor locus (Soll \& Berg, I969; Soll, I974).

Duplication of genetic material is generally associated with instability and it is therefore of interest that a number of uncharacterized suppressor genes have been described which are unstable. In bacteria, episomes have been implicated in some cases (Dawson \& Smith- 
Table I. Coprinus lagopus stocks

\begin{tabular}{|c|c|c|}
\hline Strain & Stocks & Mating type \\
\hline H5 & Wild type & $A_{5} B_{6}$ \\
\hline PR230I & $m e t-I$ & $A_{2} B_{3}$ \\
\hline MS28 & $m e t-1$ sup $-6^{+*}$ (unstable) & $A_{2} B_{3}$ \\
\hline LR4 & $m e t-I$ & $A_{5} B_{6}$ \\
\hline LRI7 & met-I sup $-3^{+}$ & $A_{5} B_{6}$ \\
\hline LR82 & met-I sup $-4^{+}$ & $A_{5} B_{6}$ \\
\hline LR72 & met-I sup- $3^{+}$sup $-4^{+}$ & $A_{5} B_{6}$ (extinct) \\
\hline
\end{tabular}

Keary, 1963; Schwartz, 1964, I965). In the fungus Aspergillus nidulans, an unstable suppressor gene mutation arose following a chromosomal translocation thought to involve a duplication (Ball, 1967). We describe an unstable suppressor in another fungus, Coprinus lagopus, which apparently involves duplication of the whole chromosome on which a suppressor locus is situated. All these unstable suppressors could be explained in terms of mutation of indispensable tRNAs.

\section{METHODS}

Stocks and symbols. The Coprinus lagopus stocks used are listed in Table I.

In the early work on gene suppression in eukaryotes, the gene symbols followed the conventional pattern, with the mutant suppressor designated sup-I, sup-2, etc., for different genes and the wild-type allele designated $\sup -I^{+}$, etc. In bacteria the $\sup -I^{+}$sign is used for the mutant (active suppressor) and $\operatorname{sup-I^{-}}$ for the wild type (inactive suppressor). In this report we have adopted the bacterial symbols, with sup $-I^{+}$as the mutant (active suppressor).

Media. Minimal (MM), complete (CM) and fruiting media have been described (Lewis, 196I). A special complete medium (SCM) was used, similar to complete medium except for the absence of casein hydrolysate and malt extract and with the addition of 0.5 p.p.m. DL-methionine.

Reversion tests, sexual crosses, somatic resolution and tip isolation. Tests for reversion of Ms28, met-I sup- $6^{+}$to auxotrophy were made with solid agar mycelial transplants taken from the growing front of the culture with a $2 \mathrm{~mm}$ cork borer and transplanted to minimal medium. Sexual crosses were made and analysed by fruiting the dikaryon and sowing the basidiospores on to complete medium with $0.01 \%$ furfuraldehyde. Somatic resolution of the two nuclear components of a dikaryon was made by isolating and re-isolating hyphal tips from long chlamydospores as described by Lewis (I96I). Hyphal tips and interstitial pieces of single hyphae were isolated by cutting $\mathrm{I} \cdot 0$ to $\mathrm{I} \cdot 5 \mathrm{~mm}$ from the tip or from an interstitial segment some 2 to $3 \mathrm{~mm}$ behind the tip.

Chromosome counts. These were obtained from germinating oidia by suspending the oidia in liquid $\mathrm{CM}$ at $37^{\circ} \mathrm{C}$ for $3 \mathrm{~h}$, centrifuging, suspending the oidia in water for $\mathrm{I} \mathrm{h}$, and evacuating. The oidia were hydrolysed in $\mathrm{I} \mathrm{M}-\mathrm{HCl}$ for $8 \mathrm{~min}$ at $60^{\circ} \mathrm{C}$, stained in Feulgen reagent for 2 to $4 \mathrm{~h}$, and gently squashed and mounted in orcein. The number of nuclei in the tip and interstitial cells was counted from similar preparations of hyphae grown on solid media. The preparations were viewed under phase-contrast microscopy. 
Table 2. Time of growth on MM plus methionine to produce reversion of stock Ms 28 from prototrophy to requiring methionine

\begin{tabular}{|c|c|c|c|}
\hline $\begin{array}{l}\text { DL-Methionine } \\
\text { (p.p.m.) }\end{array}$ & $\begin{array}{l}\text { Time to reversion } \\
\text { (h) }\end{array}$ & $\begin{array}{l}\text { DL-Methionine } \\
\text { (p.p.m.) }\end{array}$ & $\begin{array}{c}\text { Time to reversion } \\
\text { (h) }\end{array}$ \\
\hline 500 & 24 & $2 \cdot 0$ & 72 \\
\hline 200 & 24 & $I \cdot 9$ & 88 \\
\hline 100 & 48 & $1 \cdot 8$ & $72,88,100^{*}$ \\
\hline 50 & 48 & $\mathrm{I} \cdot 7$ & NR \\
\hline IO & 48 & $I \cdot 6$ & NR \\
\hline 5 & 48 & $I \cdot 5$ & NR \\
\hline 4 & 48 & L-Methionine & Time to reversion \\
\hline 3 & 48 & (p.p.m.) & (h) \\
\hline 2 & 90 & $2 \cdot 5$ & 72 \\
\hline \multirow[t]{3}{*}{ I } & NR & $I \cdot 5$ & 72 \\
\hline & & $I \cdot 0$ & 72 \\
\hline & & 0.75 & 72 \\
\hline
\end{tabular}

\section{RESULTS}

Selection for prototrophy from a met-I stock, PR230r, gave some 70 suppressed mutants (Lewis, 196I). All but one of those analysed were stable and the suppressor mutations could be assigned to one of five different loci. The one unstable suppressor strain, Ms28, readily reverted to auxotrophy if grown under conditions where the suppressor activity was not required. The simplest interpretation of this instability is that in Ms28, the suppressor locus is duplicated on an extra chromosome. The following experiments were designed to test this idea by examining the conditions which promoted the loss or retention of the sup $-6^{+}$ mutation.

\section{Stability on methionine supplemented medium}

When grown on MM, Ms28 had a normal fluffy appearance and grew slightly more slowly than a wild-type stock. When subcultured to $M M$ it remained stable indefinitely. However, when grown on CM and subcultured from the growing point by hyphal transfer, the inocula were irreversibly reverted to met-I auxotrophs by $24 \mathrm{~h}$. Supplementation of MM with methionine also caused reversion to auxotrophy.

The effect of different concentrations of methionine on reversion is shown in Table 2. At 200 p.p.m. DL-methionine reversion was complete within $24 \mathrm{~h}$, but at the threshold concentration of $\mathrm{I} \cdot 8$ p.p.m. the effect took longer and occurred after $72 \mathrm{~h}$. Below this concentration the amount of methionine present would not support growth of the met-I mutant and reversion did not occur. A concentration of methionine which supports growth of stock PR230I replaces the requirement for suppressor gene function and it was under these conditions that sup $-6^{+}$was lost from the Ms28 monokaryon.

\section{Stability in hyphal tips, segments and oidia}

Three types of colony could be derived by hyphal-tip isolations from Ms28: the unstable Ms28 type, the stable unsuppressed met- 1 , and a new type which was stable and prototrophic. This new type was not fluffy, had sparsely branched hyphae, and its growth was inhibited by 100 p.p.m. DL-methionine. All three of the above types could also be obtained from interstitial segments of some I to $2 \mathrm{~mm}$ length taken immediately behind the growing tip.

It is apparent that in the cells of the vigorous prototrophic Ms 28 three nuclear types are 
present. One gives rise to the stable prototroph which is slow growing, has an abnormal morphology and is sensitive to methionine. This type must carry the sup- $\sigma^{+}$mutation, and since it is stable, must also be haploid. The stable auxotrophic type is also haploid but has lost sup- $6^{+}$, and therefore has the sup-6 $6^{-}$wild-type allele. The ease with which either allele is lost indicates duplication of a whole chromosome rather than a smaller integrated genetic unit. If there is no selection for either allele of the sup-6 gene, these two haploid types are expected to arise by random loss of either of the duplicated chromosomes.

\section{Stability in heterozygous dikaryons}

The stability of sup $-6^{+}$was also tested under heterozygous conditions. This was possible by forming dikaryons between Ms 28 and a variety of other strains, all of which carried the wild-type allele of sup $-6^{+}$. Recovery of sup $-6^{+}$was attempted at two stages, (i) by somatic resolution of the vegetative dikaryon, and (ii) from the sexual spore progeny.

\section{Chlamydospore resolution}

Dikaryons were made between Ms28 and H5 wild type, LR4 met-I, LRI7 met-I sup- $3^{+}$, LR82 met-I sup $-4^{+}$and LR72 met-r sup $-3^{+}$sup $-4^{+}$. On resolution from germinating chlamydospores, the two nuclear components were identifiable by mating type independent of any other gene markers.

From all dikaryons, the reverted auxotrophic met-I type of MS28 was readily recovered. From the dikaryon with strain LR72, which carried two other suppressor genes, the stable prototrophic type was recovered also. This prototrophic type was also obtained from dikaryons with $\mathrm{H} 5$ and LR82 after careful nurturing of large numbers of initially non-growing tips. Thus both the expected haploid nuclear types arise in the dikaryon. The unstable Ms 28 type was not obtained from any of the chlamydospores. This may be because aneuploids germinate slowly and the chlamydospore isolation technique would give preference to faster growing haploids. The dikaryons were made on CM and all but those with LR4 (which were auxotrophic) on MM also. The media appeared to have no effect on the type of component isolated from the different dikaryons.

The dikaryon between Ms28 and the unsuppressed met-I mutant LR4 was auxotrophic: sup $-6^{+}$appears recessive by this test. However, dikaryons between $\mathrm{Ms} 28$ and strains carrying either met-I sup- $3^{+}$or met-I sup- $4^{+}$were prototrophic, showing that sup- $6^{+}$does not complement either of two non-allelic suppressor mutations (see Discussion).

\section{Analysis of sexual crosses}

Dikaryons containing the Ms28 nucleus were generally infertile and fruit bodies were obtained from only two of the dikaryons described above, one with $\mathrm{H} 5$ wild type and another with the met-I sup- $3^{+}$strain LRI7. The basidiospores' viabilities in both crosses were not more than $25 \%$. The unstable sup- $6^{+}$suppressor type was not identified in any of the progeny. From both crosses $50 \%$ of the progeny were met-I auxotrophs, indicating non-segregation of sup- $6^{+}$: a normal segregation of sup- $6^{+}$in either cross would be expected to give a maximum of $25 \%$ met-I progeny.

\section{Nuclear and chromosome number}

Three germinating oidia were found with countable chromosomes and in all three the number was $13+2$ very faintly stained dots (Fig. I). One dividing nucleus in a hyphal intercalary cell was also found to be countable, with 13 chromosomes. The haploid number for C. lagopus is 12 (Lu \& Raju, 1970). The chromosomes are extremely small and difficult 


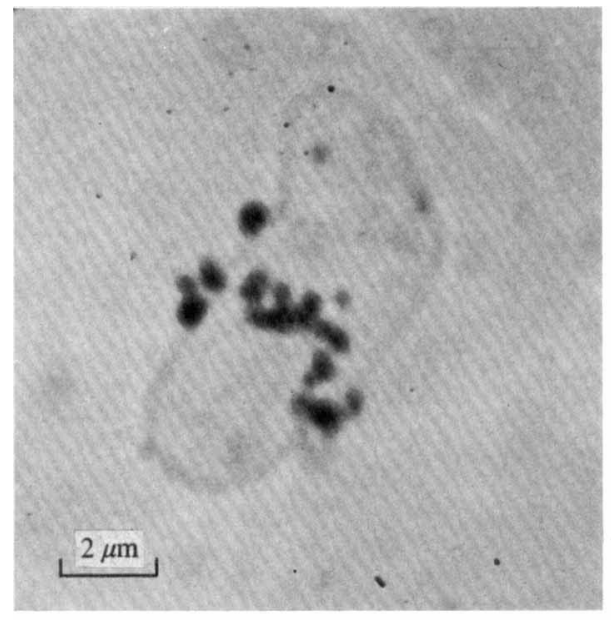

(a)

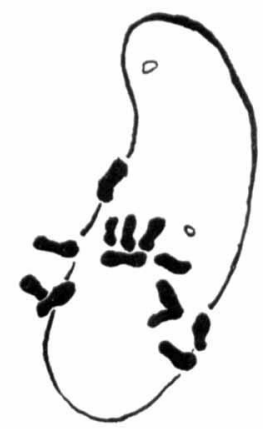

(b)

Fig. I. Germinating oidium of Ms 28 showing 13 chromosomes and 2 faintly stained spots.

to count, but it can be said that the chromosome number of Ms28 is probably 13 and is certainly more than $\mathbf{I} 2$.

Strain Ms28 differed from normal monokaryons in the number of nuclei per cell. Normal monokaryons have one, two, or (rarely) four nuclei in the tip cell and one nucleus in the interstitial cells. When grown on CM (i.e. stable auxotrophic type) Ms 28 had up to ten nuclei in the tip and two nuclei in more than $90 \%$ of the interstitial cells. The unbranching tips of the auxotrophic type were abnormally long, an outward expression of the multinuclear tip.

\section{DISCUSSION}

To interpret our results, we assume that the sup $-6^{+}$mutation occurs in a tRNA gene. This is based on the fact that three stable suppressors of met-I, sup- $3^{+}$, sup $-4^{+}$and sup $-5^{+}$ are thought to be tRNA mutants. sup $-3^{+}$and sup $-5^{+}$are allele-specific in activity (Shahriari $\&$ Casselton, 1973) and all three suppressors have the important properties of being recessive but failing to complement when combined in pairs in heterozygous dikaryons (Lewis, I96I). Although its allele specificity could not be tested, the present study shows that sup- $6^{+}$also appears recessive and non-complementing when tested in dikaryons. Todd \& Casselton (1973) have presented evidence to show that recessiveness and non-complementation are predictable properties of allele-specific missense suppressors in fungi involving tRNA structural gene mutations. We conclude that mutation to at least four different tRNAs can lead to translational correction of the met-I mutation in stock PR230I. The instability of the sup $-6^{+}$phenotype and evidence that it arises in conjunction with a chromosome duplication suggest that this mutation, unlike the other suppressors, alters an indispensable tRNA. For normal growth the mutation can only be maintained in a cell in which the sup- $6^{-}$gene is also present. (Dominance of sup- $6^{+}$in this situation is due to only part of the genome being duplicated, see Todd \& Casselton, 1973.) Whilst not completely lethal in a haploid cell, the sup- $6^{+}$is clearly detrimental to growth.

The sup- $6^{+}$mutation is readily lost from the met-I strain which carries it. Aneuploid nuclei are at a disadvantage in fungi and therefore haploidization will be favoured. Either 
chromosome will be lost at random and indeed, both haploid types were recovered from hyphal tips of the Ms 28 monokaryon grown on MM. Selection against sup $-6^{+}$haploid nuclei would be expected on SCM because suppressor activity is no longer required and the mutation depresses growth. Both types of haploid nuclei arose in cells of dikaryons formed between Ms 28 and other strains, as shown by chlamydospore resolution. In all cases the other nucleus in the dikaryon carried the sup-6- gene and therefore the semi-lethality of the suppressor mutation should not have influenced survival of the chromosome carrying it. The fact that it was difficult to recover the sup- $6^{+}$haploid type and aneuploid types may only reflect the difficulty such chlamydospores have in germinating.

The vigorous growth of the disomic sup- $6^{+}$strain and the poor growth and sensitivity to methionine of the sup- $6^{+}$haploid provide a striking analogy with strains of $E$. coli carrying the semi-lethal $s u-36^{+}$missense suppressor. This analogy provides an explanation for the indispensability of a tRNA mutation. $s u 36^{+}$arises in the only gene for a glycine tRNA which translates the GGA codon. Mutation leads to translation of the AGA arginine codon and there is no longer tRNA in the cell to translate GGA (Yanofsky, Helinski \& Maling, 196I; Hill et al. 1969; Carbon, Squires \& Hill, 1970). Healthy suppressed strains are heterogenotes in which the suppressor locus is duplicated so that both the mutant and non-mutant tRNAs are synthesized. Haploid $s u 36^{+}$cells are slow-growing and sensitive to enriched medium.

Indispensability of tRNA is perhaps surprising in fungi, because considerable redundancy is expected. Some 400 genes for tRNA exist in yeast compared with only 40 to 80 in bacteria (see Smith, 1972). Suppressor studies in yeast have shown that there are at least eight genes for tyrosine tRNA (Gilmore, Stewart \& Sherman, I968), all of which may be mutated to translate the UAA codon. However, the present study suggests that, as in bacteria, redundancy does not necessarily affect all tRNAs required by the cell. The study of unstable suppressors in fungi is an approach to understanding why such indispensable-tRNA species exist.

We are grateful to Dr J. W. Cowan and Mr D. Walls for valuable help.

\section{REFERENCES}

Altman, S., Brenner, S. \& SMIth, J. D. (I971). Identification of an Ochre-suppressing anticodon. Journal of Molecular Biology 56, 195-I97.

BALl, C. (1967). Chromosomal instability related to gene suppression in Aspergillus nidulans. Genetical Research 10, $173-183$.

Carbon, J., Squires, C. \& Hill, C. W. (1970). Glycine transfer RNA of Escherichia coli. II. Impaired GGA recognition in strains containing a genetically altered transfer RNA; reversal by a secondary suppressor mutation. Journal of Molecular Biology 52, 57I-584.

Dawson, G. W. P. \& Smith-Keary, P. E. (1963). Episomic control of mutation in Salmonella typhimurium. Heredity $18, \mathrm{I}-20$.

Gilmore, R. A., Stewart, J. W. \& Sherman, F. (I968). Amino acid replacement resulting from super-

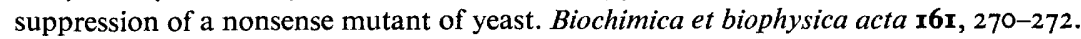

Goodman, H. M., Abelson, J. N., Landy, A., Brenner, S. \& Smith, J. D. (I968). Amber suppression: a nucleotide change in the anticodon of a tyrosine transfer RNA. Nature, London 217, 1019-1024.

GorinI, L. (1970). Suppression. Annual Review of Microbiology 4, 107-1 34.

Hill, C. W., Foulds, J., Soll, L. \& BerG, P. (1969). Instability of a missense suppressor resulting from a duplication of genetic material. Journal of Molecular Biology 39, 583-59I.

HIRSH, D. (I97I). Tryptophan transfer RNA as the UGA suppressor. Journal of Molecular Biology 58, 439-458. 
LEWIS, D. (I96I). Genetical analysis of methionine suppressors in Coprinus. Genetical Research 2 , I4I-I 55.

LU, B. C. \& RaJu, N. B. (1970). Meiosis in Coprinus. II. Chromosome pairing and lampbrush diplotene stage of meiotic prophase. Chromosoma 29, 305-316.

Riddle, D. L. \& CARBon, J. (1973). Frameshift suppression: a nucleotide addition in the anticodon of a glycine transfer RNA. Nature New Biology 242, 230-234.

Schwartz, N. M. (1964). Suppression of a lac $0^{\circ}$ mutation in Escherichia coli. Journal of Bacteriology 88, 996-100I.

SCHWARTZ, N. M. (1965). Genetic instability in Escherichia coli. Journal of Bacteriology 89, 71 2-717.

ShaHRIARI, H. \& CASSElton, L. A. (1973). By-pass and translational suppressors of methionine mutants in the fungus Coprinus lagopus. Heredity 3r, I37.

Sмiтн, J. D. (1972). Genetics of transfer RNA. Annual Review of Genetics 6, 235-256.

SMith, J. D. (I973). Genetics and structural analysis of transfer RNA. British Medical Bulletin 29, $220-225$.

Soll, L. (1974). Mutational alterations of tryptophan specific transfer RNA that generate translation suppressors of the UAA, UAG and UGA nonsense codons. Journal of Molecular Biology 86, 233-243.

Soll, L. \& BerG, P. (I969). Recessive lethal nonsense suppressor in Escherichia coli which inserts glutamine. Nature, London 223, 1340-1342.

Todd, N. K. \& CAsselton, L. A. (1973). Non-complementation and recessiveness as properties of missense suppressor genes in the fungus Coprinus. Journal of General Microbiology 77, 197-207.

Yaniv, M., FolK, W. R., Berg, P. \& Soll, L. (I974). A single mutational modification of a tryptophanspecific transfer RNA permits aminoacylation by glutamine and translation of the codon UAG. Journal of Molecular Biology 86, 245-260.

YANOFSky, C., Helinski, D. R. \& Maling, B. D. (I96I). The effects of mutation on the composition and properties of the A protein of Escherichia coli tryptophan synthetase. Cold Spring Harbor Symposia on Quantitative Biology 26, I I-23. 